\title{
INTRODUCTION \\ Formation, growth, and rupture: the biology and physics of cerebral aneurysms
}

\author{
Charles J. Prestigiacomo, MD, ${ }^{1}$ Matthew J. Gounis, PhD, ${ }^{2}$ L. Fernando Gonzalez, MD, ${ }^{3}$ and \\ Juhana Frösen, MD, $\mathrm{PhD}^{4}$
}

1Department of Neurosurgery, University of Cincinnati, Cincinnati, Ohio; ${ }^{2}$ Division of Neuroimaging and Intervention, and New England Center for Stroke Research, Department of Radiology, University of Massachusetts, Worcester, Massachusetts; ${ }^{3}$ Department of Neurosurgery, Duke University, Durham, North Carolina; and ${ }^{4}$ Department of Neurosurgery, Kuopio University, Kuopio, Finland

$\mathrm{T}$ HE rupture of a cerebral aneurysm is a devastating event for the patient and the patient's family. Despite the numerous advances in microsurgical and endovascular treatment since the early days of Dott and Dandy, the mortality and morbidity rates from aneurysmal rupture remain unacceptably high. With a mortality rate of approximately 50\% and an additional morbidity rate of $25 \%$, almost 3 in 4 patients sustaining a subarachnoid hemorrhage never return to their pre-ictal baseline. Additionally, though the microsurgical and endovascular management of intracranial aneurysms has enjoyed a substantial improvement in outcomes, these procedures nonetheless carry a significant complication rate.

Given the availability of noninvasive neuroimaging, unruptured aneurysms are being incidentally found at unprecedented rates. It is thus imperative that, to maximize the good outcomes for patients with aneurysms, one must first identify which unruptured aneurysm has a significant risk of rupture. The goal must be to expose only those patients having aneurysms at a high risk of rupture to the potential complications of treatment and, concomitantly, to diagnose stable aneurysms that should be conservatively followed. Identifying criteria that will provide the clinician this ideal algorithm remains critically important for optimal outcomes, as well as to reduce unnecessary costs in healthcare.

Science and technology have been instrumental in providing researchers from seemingly disparate fields the tools to help unravel the biology, mathematics, and physics of aneurysm initiation, growth, rupture, and recurrence. Over the last 20 years, the body of literature discussing cerebral aneurysms and their formation and rupture has expanded to incorporate contributions from geneticists, molecular biologists, mathematicians, and engineers. These contributions to scientific knowledge serve to aid clinicians with evidence to guide treatment and/or conservative surveillance options. As the science continues to unravel the mysteries of aneurysm initiation, growth, and rupture, there may ultimately be a personalized medicine approach to preventing rupture and certainly to tailoring optimum treatment to each patient.

This issue of Neurosurgical Focus addresses several of the important biological and genetic aspects of aneurysm formation. The reader will be exposed to the latest theories of aneurysm formation that invoke genetic predispositions and the role of inflammation and matrix metalloproteinases in the genesis of a cerebral aneurysm. Observational studies provide additional insight into the role of estrogen and other female sex steroids and tobacco use with respect to the evolution of multiple aneurysms.

Morphometric and hemodynamic relationships can provide vast amounts of information, especially in this era of powerful computer algorithms. Combined with the tremendous growth of the field of computational fluid dynamics analysis and computational modeling, the reader will recognize how much information can be gleaned through the simple power of observational analysis.

Imaging's explosive evolution now enables clinicians to assess inflammation of aneurysm walls, which may help determine rupture risk, and many researchers in laboratories throughout the world continue to aggressively pursue in vivo models to help provide answers that no other methodologies can provide.

As with all growing fields of study, this field is not without controversy. Computer-based modeling of aneurysms and fluid dynamics analysis of an aneurysm have yet to be 
definitively validated against experimental methods. Are the formulae that guide the analysis accurate and precise? Do they reflect the true nature of the forces and flows that occur in aneurysms and their parent vessels? Which hemodynamic parameters are relevant? Indeed, most models of aneurysms lack the necessary modeling of the vessel wall, and more importantly, we lack the ability to understand how the anisotropic mechanics of specific segments of an aneurysm wall influence the behavior of that aneurysm with respect to rupture probability.

Understanding aneurysms and their behavior is clearly a herculean task that, as noted, requires the passion, energy, and talents of many specialists in fields that may not necessarily be traditionally associated with the field of neurosurgery. It also requires the sharing of data from around the world, a task that can only be achieved through international cooperation. Though this issue of Neurosurgical Focus will provide several answers to the many questions of aneurysmal behavior, it will likely engender many more questions and challenges from the readership. Thus, this issue will serve its dual purpose: It shall inform the readership and help organize, in part, the current stateof-the-art in this field, and it shall stimulate the readership to more deeply explore the many areas that remain unexplored.

https://thejns.org/doi/abs/10.3171/2019.4.FOCUS19333

\section{Disclosures}

The authors report no conflict of interest.

\section{Correspondence}

Charles J. Prestigiacomo: presticj@ucmail.uc.edu. 